\title{
ACCURACY AND PRECISION OF FLASH GLUCOSE MONITORING SENSORS INSERTED INTO THE ABDOMEN AND UPPER THIGH COMPARED TO THE UPPER ARM (OUT OF SIGHT)
}

Running title: FGM performance on the arm, abdomen, and thigh Sara Charleer ${ }^{1,2}$, Chantal Mathieu $^{1}$, Frank Nobels $^{3}$, Pieter Gillard $^{1}$

${ }^{1}$ Department of Endocrinology, University Hospitals Leuven - KU Leuven, Leuven, Belgium.

${ }^{2} \mathrm{PhD}$ fellowship strategic basic research of the Research Foundation - Flanders (FWO), Brussels, Belgium.

${ }^{3}$ Department of Endocrinology, OLV Hospital Aalst, Aalst, Belgium.

Correspondence to: Pieter Gillard, UZ Leuven, Herestraat 49, 3000 Leuven, Belgium. Tel.: +32 163406 15, Fax: +32 163469 89, Email: pieter.gillard@uzleuven.be NCT03154060, ClinicalTrials.gov

\begin{abstract}
Nowadays, most Belgian patients with type 1 diabetes use flash glucose monitoring (FreeStyle ${ }^{\circledR}$ Libre $\left.^{\mathrm{TM}}[\mathrm{FSL}]\right)$ to check their glucose values, but some patients find the sensor on the upper arm too visible. The aim of this study was to compare accuracy
\end{abstract}

This article has been accepted for publication and undergone full peer review but has not been through the copyediting, typesetting, pagination and proofreading process, which may lead to differences between this version and the Version of Record. Please cite this article as doi: 10.1111/dom.13239 
and precision of FSL sensors when placed on different sites. Twenty-three adults with type 1 diabetes used three FSL sensors simultaneously for 14 days on upper arm, abdomen, and upper thigh. FSL measurements were compared to capillary blood glucose (BG) measurements obtained with built-in FSL BG meter. Aggregated mean absolute relative difference was $11.8 \pm 12.0 \%, 18.5 \pm 18.4 \%$ and $12.3 \pm 13.8 \%$ for arm, abdomen ( $p=0.002$ vs. arm) and thigh ( $p=0.5$ vs. arm) respectively. Clarke error grid analysis for arm and thigh were comparable (zone A: $84.9 \%$ vs. $84.5 \%, p=0.6$ ), while less accuracy was seen for abdomen $(69.4 \%, p=0.01)$. Apart from the first day, accuracy of FSL sensors on arm and thigh was more stable across the 14-day wear duration than accuracy of sensors on abdomen, which deteriorated mainly during week two $(p<0.0005)$. Aggregated precision absolute relative difference was markedly lower for arm/thigh (10.9 $\pm 11.9 \%)$ compared to arm/abdomen $(20.9 \pm 22.8 \%)$ $(p=0.002)$. Our results indicate that accuracy and precision of FSL sensors placed on upper thigh are comparable to upper arm, whereas abdomen performed unacceptably poor.

\section{INTRODUCTION}

Since the introduction and full reimbursement of flash glucose monitoring in the form of the FreeStyle ${ }^{\circledR}$ Libre $^{\mathrm{TM}}$ (FSL; Abbott Diabetes Care, Alameda, CA) most Belgian patients with type 1 diabetes switched to this system. However, some patients find the sensor on the upper arm too visible. Because accuracy was only tested on the upper arm, ${ }^{1}$ product information brochure does not recommend placing the sensor on a different body site. ${ }^{2}$ 
The objective of the current study was to evaluate accuracy and precision of FSL sensors when inserted into less visible sites such as abdomen and upper thigh compared to back of the upper arm.

\section{METHODS}

2.1. Study protocol. This open-label, prospective, single-arm study was conducted at two research sites in Belgium from May 19, 2017 until August 3, 2017. Twenty-three adults with type 1 diabetes using FSL sensors on upper arm for more than three months were included. Participants wore three FSL sensors, all from the same production lot, simultaneously. Sensors were placed on back of upper arm, abdomen, and upper thigh for 14-days. Regions with lipodystrophy were only seen in two patients and were avoided as insertion site. Patients were given enough back-up sensors to immediately replace prematurely lost sensors, while the sensors on the parallel sites were not replaced. Throughout the study, patients were instructed to measure capillary blood glucose (BG) with built-in FSL BG meter at least seven times per day. Preferred testing was before each meal, two hours after each meal and at bedtime. Additionally, to obtain every measured interstitial glucose value, patients were instructed to scan each sensor with its connected FSL reader at least every eight hours.

The coordinating institutional review board (Ethics Committee University Hospital Leuven) approved the study protocol after acquiring advice from the local ethical committee. Written informed consent was obtained from every participant prior to enrolment. The study was executed in line with Good Clinical Practice guidelines of the Declaration of Helsinki in its latest form. This study is registered at ClinicalTrials.gov (NCT03154060). 
2.2. Evaluation of accuracy and precision. To compare FSL readings with paired reference BG values, aggregated mean absolute relative difference (MARD) and percentage of data points in zone $A$ and $B$ of the Clarke error grid ${ }^{3}$ were used. To assess the evolution of accuracy over the 14-day wear period, MARD per day was calculated for each insertion site. If a sensor was lost before end of study, a new sensor was inserted immediately, and subsequent paired FSL and reference capillary BG readings started again from day 1 onwards until the patient reached end of study (day 14) to allow comparison with other sites. Precision of FSL sensors across body sites was evaluated by the aggregated precision absolute relative difference (PARD) ${ }^{4}$ and by the interquartile range of individual MARD values. ${ }^{5}$ Because of a car accident, data of one patient could not be recovered for data analysis.

2.3. Statistical analysis. With a sample size of 20 patients, the study was powered to detect significant differences in MARD between arm and abdomen/thigh of $>1 \%$ with $80 \%$ power. Results are reported as median (interquartile range) or mean $\pm S D$ unless otherwise stated. Performance measures for different body sites were compared using Wilcoxon signed rank test. McNemar's test was used to analyze qualitative data across body sites. A two-tailed $p$-value with an $\alpha$-level for significance was set at 0.05 . Using Tukey fences method, we performed an outlier analysis for paired readings to discover potentially large differences between two sensors that were possibly masked, since large numbers of paired readings were used to calculate patients' PARD values. Outliers in paired sensor readings for arm/thigh and arm/abdomen were defined as: outlier = PARD $>3$ rd quartile + $\left(I Q R^{*} 1.5\right)$ for more than 1 hour. ${ }^{6}$ 


\section{RESULTS}

3.1. Patient characteristics. Of initial 23 subjects included, one patient prematurely ended the study due to a severe car accident (not caused by hypoglycemia). Of the 22 patients (12 male), 18 used multiple daily insulin injections and 4 were on insulin pump therapy (Medtronic Paradigm ${ }^{\circledR} \mathrm{Veo}^{\mathrm{TM}} 754$ [Medtronic, Northridge, CA, USA]) with the cannula inserted in the abdomen. Median (IQR) age was $57.5(40-68)$ years, BMI was $24.9(23.0-25.6) \mathrm{kg} / \mathrm{m}^{2}$, duration of diabetes was 19.5 (10.2-39.2) years and $\mathrm{HbA}_{1 \mathrm{c}}$ was $7.2(6.9-7.4) \%(55[52-57] \mathrm{mmol} / \mathrm{mol})$.

3.2. Performance evaluation. Adherence to perform at least seven capillary BG tests per day was acceptable (median 6.8/day; IQR 6.3-7.0). An overview of accuracy and precision measures can be found in table 1. Overall, MARD was lowest for sensors on arm and thigh compared with sensors on abdomen. Only in hypoglycemic range sensors performed comparably poor, with MARD values above $20 \%$ for each body site. In some subjects however, sensor values in arm and/or thigh were also accurate in hypoglycemic range, illustrated by MARD values below $10 \%$ to $13 \%$ in up to $25 \%$ of subjects (table 1 ). For each insertion site, MARD was significantly higher on day one after sensor insertion than on day two (arm: $15.0 \pm 12.9 \%$ vs. $11.1 \pm 8.7 \%$, $p=0.008$; abdomen: $17.6 \pm 16.5 \%$ vs. $13.5 \pm 10.7 \%, p=0.036$; thigh: $15.4 \pm 14.3 \%$ vs $11.6 \pm 9.4 \%, p=0.015)$. Accuracy of FSL sensors on arm and thigh was more stable over time than accuracy of sensors on abdomen, which deteriorated mainly during the second week $(p<0.0005)$ (figure 1). The observed MARD values did not change when only data of sensors worn the entire 14-day period were analyzed (data not shown). The observed MARD values did not change when data of the replaced 
sensors were not included in the analysis (data not shown). Median (IQR) MARD of insulin pump users was not different from the patients on MDI for the arm (9.3 [4.6$16.3] \%$ vs $8.7[4.2-16.0] \%)$, the abdomen (13.2 [6.4-30.7]\% vs $12.5[5.6-25.1] \%)$, and thigh $(8.6[4.2-15.3] \%$ vs $9.4[4.1-16.4] \% ; p=N S$ for all comparisons). Percentages of data points in zones $\mathrm{A}$ and $\mathrm{B}$ of the Clarke error grid were comparable for arm and thigh $(p=0.6)$, while for abdomen a lower percentage in zone $A(p=0.01)$ and thus less accuracy was observed (figure 2).

PARD was markedly lower for arm/thigh compared to arm/abdomen indicating that sensor values of arm and thigh were better matched than sensor values of arm and abdomen. For arm/thigh, a total of 122 hours of outliers were seen in the 22 patients over the whole study period. Sixty-six percent of time outliers occurred during daytime, but not during sports or more intense physical activity, such as cycling to work. Thirty-four percent of outliers occurred during the night. High number of outliers $(43 \%)$ was seen during day one of sensor wear. For arm/abdomen, outliers comprised 134 hours. Fifty-four percent of time outliers occurred during daytime and were also not correlated with sports or physical activity. Forty-six percent of outliers occurred during the night and only $17 \%$ of outliers were observed during day one of sensor wear. Interquartile ranges of individual MARD's showed the same pattern with lowest variation in arm and thigh, except in the hypoglycemic range where all insertion sites showed higher interindividual variation (table 1).

Sensors on thigh were more often lost (nine times by seven patients) than those on arm or abdomen (one time on each site; $p=0.05$ compared to thigh). More patients $(n=14)$ preferred to place FSL sensors on back of the upper arm compared to thigh $(n=6 ; p=0.8)$ and abdomen $(n=4, p=0.02)$ even though they found sensors on the arm most visible to the outside world. Local hematoma was observed in one patient when 
the sensor was placed on the abdomen and in another patient when sensor was placed on the thigh. Local hematoma was observed in one patient when the sensor was removed from the abdomen, at the end of study. Without data of this sensor, MARD of sensor on abdomen $(18.8 \pm 18.6 \%)$ was perfect in line with the data of the entire cohort so we decided not to exclude this sensor in the further analysis. A second hematoma was seen in another patient immediately after the sensor was placed on the thigh. The sensor was immediately removed, and another sensor was repositioned at a different location on the thigh, where no hematoma occurred.

\section{DISCUSSION}

This study is the first to compare performance of FSL sensors between three different sites in subjects with long standing type 1 diabetes. It shows that FSL sensors inserted into the upper thigh have acceptable accuracy as compared to sensors placed on the upper arm, while acceptable accuracy could not be demonstrated for sensors inserted into the abdomen.

Accuracy of FSL sensors on back of the upper arm was in line with previous reported data. ${ }^{1,7-10}$ To date, only one study reported accuracy of FSL readings when sensors were inserted into a different site than upper $\mathrm{arm}^{7}$ That study, performed in 7 patients with type 1 diabetes and 11 patients with type 2 diabetes, showed less accuracy of sensors placed on abdomen compared to upper arm. The current study extends this finding to a larger group of patients with type 1 diabetes confirming that insertion of FSL sensors into the abdomen should definitely be discouraged in patients with type 1 diabetes. This is in contrast to non-factory calibrated sensors which are recommended to be placed on the abdomen and can also be inserted in 
arm and gluteus without accuracy loss. ${ }^{5}$ For sensors such as Dexcom® G4/5 (Dexcom, Inc, San Diego, CA, USA) and Medtronic Enlite (Medtronic, Northridge, CA, USA) recalibration is critical to maintain accuracy over wear time. ${ }^{11}$ Possible local differences between insertion sites, such as fat distribution and motion, are taken into account by in vivo (re)calibration. Although aggregated in vivo sensitivities of a factory calibrated wired-enzyme sensor were shown to be comparable between arm and abdomen ${ }^{12}$, exact evolution of FSL MARD values over 14 days has not been studied for the abdomen before. We observed a clear deterioration of MARD values over time for the abdomen where biomechanical factors, such as pressure and motion, can alter tissue physiology around the sensor and thus sensor performance. ${ }^{13}$ Accuracy of FSL sensors on arm and thigh was stable over time apart from a lower first-day accuracy, which is caused by local tissue damage when inserting the sensor. ${ }^{1,14}$

To the best of our knowledge, this is the first time that performance of FSL sensors was evaluated when inserted into the upper thigh. Our data show that FSL sensors on thigh have acceptable accuracy, hence this site might be suggested as alternative for the upper arm in patients who find the latter site too visible. It should be explained to patients however that the upper thigh is associated with more frequent sensor loss. Future studies could be designed to examine if another site such as lateral lower back could serve as a good alternative with acceptable accuracy and low frequency of sensor loss.

Sensor-to-sensor precision, as measured by PARD, agreed with MARD, indicating that sensors on arm and thigh traced close together, while sensors on abdomen did 
not. However, both for thigh and abdomen, periods of larger differences in paired sensor readings were observed during day and night. Differences during daytime are possibly related to motion, pressure, and rubbing by clothes while differences during the night could be caused by lying directly on the sensor. Local pressure induces temporary reduction of blood flow through the insertion site resulting in a drop of sensor signal ${ }^{13}$ and consequently aberrant glucose values.

As in other studies, ${ }^{7,15}$ accuracy and precision within hypoglycemic range was markedly lower than in euglycemic and hyperglycemic ranges for all insertion sites tested. However, in some subjects sensor values were also accurate in lower ranges. It is currently not known if this is a consequence of differences between sensors of the same lot or differences between patients. Until performance of sensors in hypoglycemic range is improved, it remains important to advise patients to measure capillary BG in case of doubt, irrespectively of the site used.

This study has limitations. First, FSL sensor accuracy was evaluated against capillary instead of Yellow Springs Instruments (YSI, Yellow Springs, OH) BG readings. Because this issue affects all insertion sites equally and capillary BG readings provide more reference points, the impact on conclusions relevant for daily patient use is expected to be minimal. Second, due to inherent inability of the software to collect FSL glucose trend information, this valuable information could not be compared between different insertion sites. Finally, the present study was performed with a single sensor at each site. Although data are in line with the study of Fokkert et al, ${ }^{7}$ placing two sensors at each site could indicate if discrepancies in paired glucose values are sustained or not. 
In conclusion, this is the first study to report performance comparisons for FSL sensors on three different body sites. Findings indicate that the upper thigh can be a reasonable, more discrete site for sensor insertion, when measures are taken to avoid sensor loss.

\section{ACKNOWLEDGMENTS}

This study is accepted as an oral presentation for the 11th International Conference on Advanced Technologies \& Treatments for Diabetes (ATTD 2018) in Vienna, Austria, 14-17 February 2018.

Funding. This study was supported by the European Institute of Innovation \& Technology (EIT).

Conflict of Interest. The authors declare that there is no conflict of interest associated with this manuscript.

Author Contributions. SC contributed to the study design, implemented the study protocol, collected and analyzed the data, interpreted the data, wrote the first draft of the manuscript and contributed to subsequent revisions, and contributed to intellectual content. CM conceived and contributed to the study design, interpreted the data, reviewed the drafts of the manuscript, and contributed to intellectual content. FN contributed to the study design, oversaw the implementation of the study protocol in OLV Hospital Aalst, interpreted the data, reviewed the drafts of the manuscript, and contributed to intellectual content. PG conceived and designed the study, oversaw the study implementation in University Hospital Leuven, interpreted the data, contributed to the writing of the first draft of the manuscript and wrote subsequent revisions, and contributed to intellectual content. PG is the guarantor of 
this work and, as such, had full access to all the data in the study and takes responsibility for the integrity of the data and the accuracy of the data analysis.

\section{REFERENCES}

1. Bailey T, Bode BW, Christiansen MP, Klaff LJ, Alva S. The Performance and Usability of a Factory-Calibrated Flash Glucose Monitoring System. Diabetes Technol Ther. 2015;17(11):787-794.

2. Abbott Diabetes Care. User's Manual FreeStyle Libre Flash Glucose Monitoring System. https://freestylediabetes.co.uk/images/uploads/documents/FreeStyle_Libre_Ma nual.pdf. Accessed November 28, 2017.

3. Wentholt IME, Hart AAM, Hoekstra JBL, Devries JH. How to assess and compare the accuracy of continuous glucose monitors? Diabetes Technol Ther. 2008;10(2):57-68.

4. Obermaier K, Schmelzeisen-Redeker G, Schoemaker M, et al. Performance evaluations of continuous glucose monitoring systems: precision absolute relative deviation is part of the assessment. J Diabetes Sci Technol. 2013;7(4):824-832.

5. Faccioli S, Del Favero S, Visentin R, et al. Accuracy of a CGM Sensor in Pediatric Subjects With Type 1 Diabetes. Comparison of Three Insertion Sites: Arm, Abdomen, and Gluteus. J Diabetes Sci Technol. 2017;11(6):1147-1154.

6. Tukey JW. Exploratory Data Analysis. Massachusetts: Addison-Wesley; 1977.

7. Fokkert MJ, van Dijk PR, Edens MA, et al. Performance of the FreeStyle Libre Flash glucose monitoring system in patients with type 1 and 2 diabetes mellitus. BMJ open diabetes Res care. 2017;5(1):e000320. 
8. Ji L, Guo X, Guo L, Ren Q, Yu N, Zhang J. A Multicenter Evaluation of the Performance and Usability of a Novel Glucose Monitoring System in Chinese Adults With Diabetes. J Diabetes Sci Technol. 2017;11(2):290-295.

9. Olafsdottir AF, Attvall S, Sandgren U, et al. A Clinical Trial of the Accuracy and Treatment Experience of the Flash Glucose Monitor FreeStyle Libre in Adults with Type 1 Diabetes. Diabetes Technol Ther. 2017;19(3):164-172.

10. Aberer F, Hajnsek M, Rumpler M, et al. Evaluation of subcutaneous glucose monitoring systems under routine environmental conditions in patients with type 1 diabetes. Diabetes Obes Metab. 2017;19(7):1051-1055.

11. Wisniewski N, Moussy F, Reichert WM. Characterization of implantable biosensor membrane biofouling. Fresenius J Anal Chem. 2000;366(6-7):611621.

12. Hoss U, Budiman ES, Liu H, Christiansen MP. Feasibility of Factory Calibration for Subcutaneous Glucose Sensors in Subjects With Diabetes. J Diabetes Sci Technol. 2014;8(1):89-94.

13. Helton KL, Ratner BD, Wisniewski NA. Biomechanics of the sensor-tissue interface-effects of motion, pressure, and design on sensor performance and foreign body response-part II: examples and application. J Diabetes Sci Technol. 2011;5(3):647-656.

14. Kenneth Ward W. A review of the foreign-body response to subcutaneouslyimplanted devices: the role of macrophages and cytokines in biofouling and fibrosis. J Diabetes Sci Technol. 2008;2(5):768-777.

15. Freckmann G, Pleus S, Link M, Zschornack E, Klotzer H-M, Haug C. Performance evaluation of three continuous glucose monitoring systems: comparison of six sensors per subject in parallel. J Diabetes Sci Technol. 
2013;7(4):842-853.

FIGURE 1. Day by day evolution of mean absolute relative difference (MARD) of FreeStyle $\circledast$ Libre $^{\mathrm{TM}}$ (FSL) flash glucose monitoring sensors in the arm, abdomen, and thigh in patients with type 1 diabetes. Data points represent mean (SE) of MARD per day of sensor wear.

$M A R D=$ Mean Absolute Relative Difference, FSL=FreeStyle ${ }^{\circledR}$ Libre $^{\mathrm{TM}}, \mathrm{SE}=\mathrm{Standard}$ Error.

${ }^{* * *}$ denotes $p<0.001,{ }^{* *}$ denotes $p<0.01$, and ${ }^{*}$ denotes $p<0.05$ compared to value of the arm (for MARD). $\dagger$ denotes a significant difference of MARD over time for sensors inserted into the abdomen. $\S$ denotes a significant difference in MARD between day 1 and day 2 for all insertion sites. 


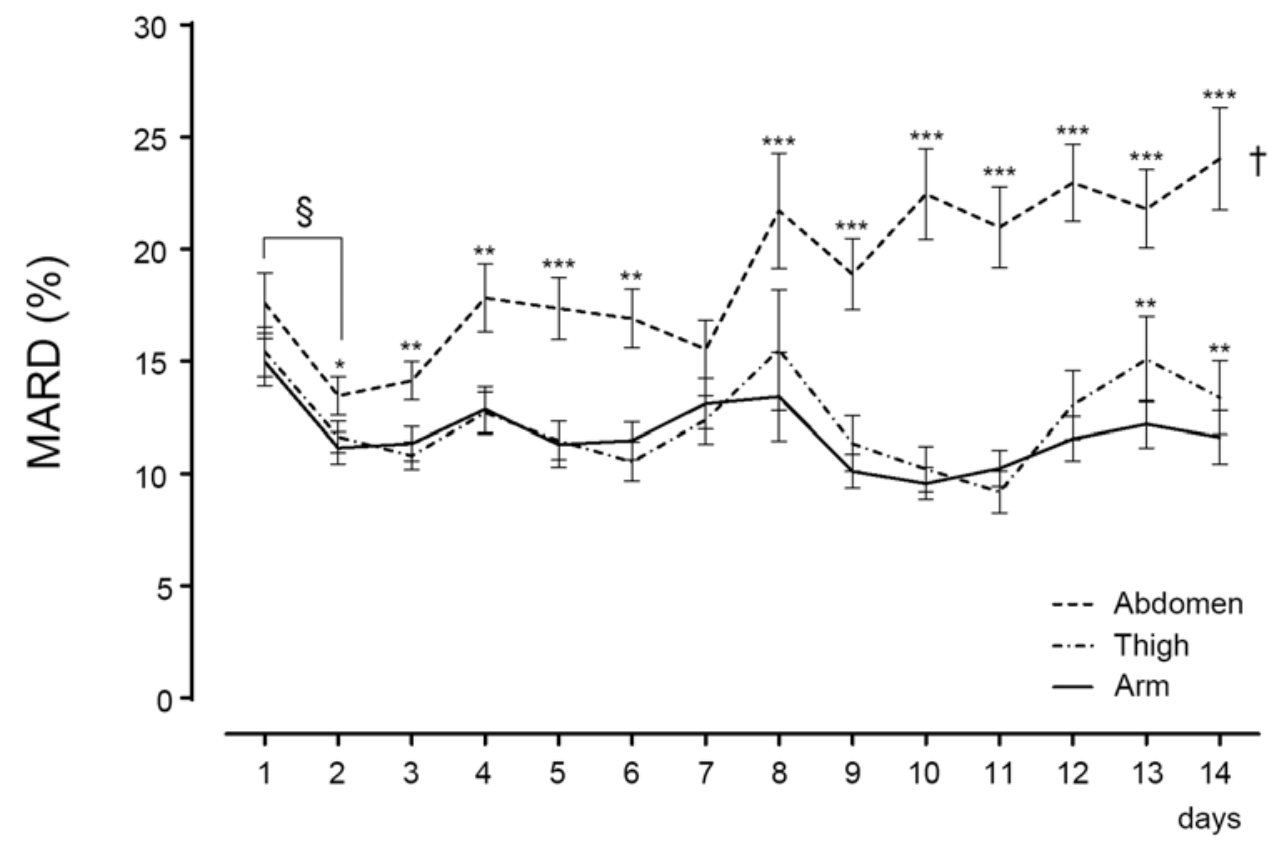

FIGURE 2. Clarke error grid analysis for FreeStyle ${ }^{\circledR}$ Libre ${ }^{\mathrm{TM}}$ flash glucose monitoring sensors in the arm (a), abdomen (b), and thigh (c) in patients with type 1 diabetes. Number and percentages of paired readings within Clarke error grid zones A, B, C D and $\mathrm{E}$ are presented. $\mathrm{FSL}=$ FreeStyle ${ }^{\circledR}$ Libre $^{\mathrm{TM}}, \mathrm{BG}=\mathrm{Blood}$ Glucose. 


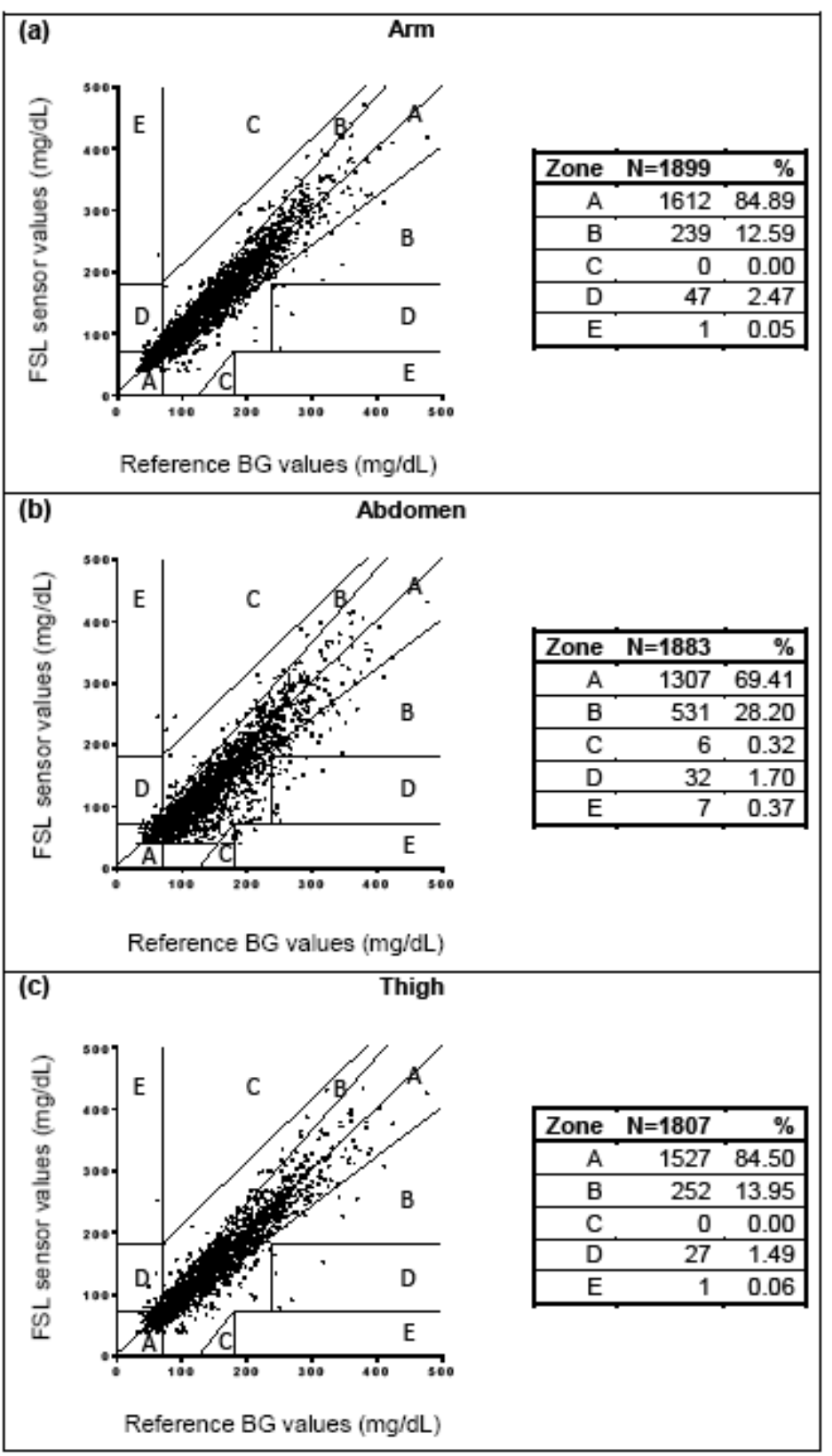


TABLE 1. Glucose sensor accuracy and precision assessed for FreeStyle ${ }^{\circledR}$ Libre $^{\mathrm{TM}}$ flash glucose monitoring sensors in the arm, abdomen, and thigh in patients with type 1 diabetes

\begin{tabular}{|l|c|c|c|c|c|}
\hline \multirow{2}{*}{} & \multicolumn{3}{|c|}{ MARD (\%) } & \multicolumn{2}{c|}{ PARD (\%) } \\
\cline { 2 - 6 } & Arm & Abdomen & Thigh \\
$(n=1,899)$ & $(n=1,883)$ & Arm vs abdomen & $\begin{array}{c}\text { Arm vs thigh } \\
(n=1,807)\end{array}$ \\
$(n=28,465)$ & $27,336)$ \\
\hline $40-500 \mathrm{mg} / \mathrm{dL}$ & $11.8 \pm 12.0$ & $18.5 \pm 18.4^{* *}$ & $12.3 \pm 13.8$ & $20.9 \pm 22.8$ & $10.9 \pm 11.9^{* *}$ \\
$(2.2-27.8 \mathrm{mmol} / \mathrm{L})$ & $(9.2-13.8)$ & $(11.3-24.0)$ & $(9.7-15.3)$ & & \\
\hline$\leq 70 \mathrm{mg} / \mathrm{dL}$ & $22.2 \pm 25.6$ & $22.2 \pm 26.9$ & $20.6 \pm 32.0$ & $19.0 \pm 16.5$ & $14.9 \pm 15.4^{* * *}$ \\
$(3.9 \mathrm{mmol} / \mathrm{L})$ & $(13.3-23.9)$ & $(12.6-26.1)$ & $(10.2-20.3)$ & & $12.0 \pm 13.0^{* * *}$ \\
\hline $70-180 \mathrm{mg} / \mathrm{dL}$ & $11.4 \pm 9.7$ & $19.5 \pm 18.6^{* *}$ & $12.3 \pm 10.9$ & $23.7 \pm 25.0$ & \\
$(3.9-10.0 \mathrm{mmol} / \mathrm{L})$ & $(9.2-15.0)$ & $(10.4-26.7)$ & $(9.3-14.8)$ & & $8.0 \pm 7.9^{* * *}$ \\
\hline$\geq 180 \mathrm{mg} / \mathrm{dL}$ & $9.5 \pm 7.9$ & $15.5 \pm 14.4^{*}$ & $9.8 \pm 8.7$ & $15.7 \pm 18.2$ & \\
$(10.0 \mathrm{mmol} / \mathrm{L})$ & $(8.2-11.5)$ & $(8.3-18.5)$ & $(7.0-12.5)$ & & \\
\hline
\end{tabular}

The number of values $(n)$ provided is the number of individual paired glucose readings that were included in the evaluation. Displayed are mean and standard deviation calculated from these individual pairings. ${ }^{\dagger}$ Value between brackets shows the interquartile range of patientspecific MARD. ${ }^{* * *}$ denotes $p<0.001,{ }^{* *}$ denotes $p<0.01$, and ${ }^{*}$ denotes $p<0.05$ compared to value of the arm (for MARD) or compared to value of arm vs abdomen (for PARD). MARD=Mean Absolute Relative Difference, PARD=Precision Absolute Relative Difference. 